\title{
Non-invasive cardiac output measurement by electrical cardiometry and M-mode echocardiography in the neonate: a prospective observational study of 136 neonatal infants
}

\author{
Su-Hua Xu ${ }^{1}$, Jing Zhang ${ }^{2}$, Yi Zhang ${ }^{3}$, Peng Zhang ${ }^{1}$, Guo-Qiang Cheng ${ }^{1}$ \\ ${ }^{1}$ Neonatology Department, Children's Hospital of Fudan University, Shanghai, China; ${ }^{2}$ Heart Center, Children's Hospital of Fudan University, \\ Shanghai, China; ${ }^{3}$ Department of Clinical Epidemiology, Children's Hospital of Fudan University, Shanghai, China \\ Contributions: (I) Conception and design: SH Xu, J Zhang, GQ Cheng; (II) Administrative support: GQ Cheng; (III) Provision of study materials or \\ patients: J Zhang, GQ Cheng, P Zhang; (IV) Collection and assembly of data: SH Xu, J Zhang, P Zhang; (V) Data analysis and interpretation: SH \\ Xu, J Zhang, Y Zhang; (VI) Manuscript writing: All authors; (VII) Final approval of manuscript: All authors. \\ Correspondence to: Guo-Qiang Cheng, PhD. Neonatology Department, Children's Hospital of Fudan University, 399 Wan Yuan Road, Shanghai \\ 201102, China. Email: gqcheng_cm@fudan.edu.cn.
}

\begin{abstract}
Background: Electrical cardiometry (EC) is a continuous, non-invasive method for measuring cardiac output (CO). This study investigates the correlation and consistency of $\mathrm{CO}$ values in newborns obtained by using EC and M-mode echocardiography (Teichholz formula).

Methods: In this prospective observational study, simultaneous measurement of CO was implemented with EC (COec) and M-mode echocardiography (COm) in neonates. The absolute values of CO measured by the two methods were converted to Z-scores. Following that, Pears's correlation analyses and the Bland-Altman index were employed to analyze the correlation and consistency of COec Z-scores and COm Z-scores.

Results: A total of 136 neonates (93 preterm infants) were enrolled in this study, and EC and M-mode echocardiography comparative studies were conducted 155 times. The mean value of COec and COm demonstrated significant statistical differences $(\mathrm{P}<0.001)$. A moderate correlation $(\mathrm{r}=0.601 ; \mathrm{P}<0.001)$ was found between the two methods. The Bland-Altman index value was 3.2\%, which remained less than $5 \%$ in the low birth weight (LBW) (2.1\%), non-LBW (3.4\%), spontaneous respiration $(3.1 \%)$, nasal continuous positive airway pressure (nCPAP) $(4.0 \%)$, mechanical ventilation $(2.9 \%)$, hemodynamic significance of the patent ductus arteriosus (hsPDA) (4.3\%), and non-hsPDA (3.7\%) groups, respectively.

Conclusions: Although the absolute values of $\mathrm{CO}$ measured by EC and M-mode echocardiography were not interchangeable, the distribution of $\mathrm{CO}$ in EC and M-mode echocardiography was similar.
\end{abstract}

Keywords: Cardiac output (CO); electrical cardiometry (EC); M-mode; neonatal infants; transthoracic echocardiography (TTE)

Submitted Jan 13, 2021. Accepted for publication Jun 10, 2021.

doi: $10.21037 / \mathrm{tp}-21-20$

View this article at: https://dx.doi.org/10.21037/tp-21-20

\section{Introduction}

Accurate and continuous measurement of cardiac output (CO) is an important part of evaluating the cardiovascular status in newborns, especially in patients with unstable hemodynamics. However, pulse profilometry, Fick's technique, and pulmonary artery catheter's thermodilution, while considered acceptable for measuring $\mathrm{CO}$, are invasive and much more traumatic for neonates (1).

Electrical cardiometry (EC), based on thoracic electrical bioimpedance, can measure CO continuously and noninvasively. The EC's working principle of estimation of $\mathrm{CO}$ is to utilize changes in thoracic electrical impedance, which is mainly influenced by erythrocyte orientation and peak flow velocity in the ascending aorta throughout the 
cardiac cycle. Before the aortic valve opening, erythrocytes are randomly orientated, resulting in increased impedance and decreased conductance. However, erythrocytes align in a parallel fashion with pulsatile blood flow, which results in less impedance and greater conductivity. The difference in thoracic conductance during the cardiac cycle allows for the calculation of $\mathrm{CO}(2)$.

Transthoracic echocardiography (TTE), as a noninvasive method to assess $\mathrm{CO}$, has been widely used in clinical practice despite being noncontiguous and technically demanding for echocardiographic technicians (3). Several common qualitative measurements can be used to estimate the stroke volume of the left ventricular (LV), mainly including the Simpson formula, Teichholz formula, the product of trans-aortic valve flow velocity time integral (VTI), and aortic valvar area (4). Although using color Doppler to measure VTI is the most common method in most neonatal and pediatric studies, it is limited to aligning with the LV outflow, placing the sample volume, and measuring the $\mathrm{LV}$ outflow tract area (5). The correlation and accuracy of $\mathrm{CO}$ values measured with EC and TTE (via VTI) have been reported in not only full term $(6,7)$ but also preterm newborns $(8-13)$. In both children and newborns, the percentage shortening fraction and ejection fraction (based on the measurement of $\mathrm{LV}$ dimensions in diastole and systole by M-mode) are the general parameters to assess $\mathrm{LV}$ systolic function $(4,5)$.

Moreover, M-mode echocardiography (Teichholz formula) has higher temporal resolution and can be easily performed with any echocardiographic system (5). For the reasons above, M-mode echocardiography is the routine method for CO measurement in The Children's Hospital of Fudan University (our hospital). However, there have been few studies on its correlation and consistency with EC. Therefore, this study explores the correlation and consistency between EC and M-mode echocardiography in neonates. We present the following article in accordance with the STROBE reporting checklist (available at https:// dx.doi.org/10.21037/tp-21-20).

\section{Methods}

\section{Participants}

This prospective observational study was conducted in the Neonatal Department of The Children's Hospital of Fudan University (our hospital) from April 2018 to December 2018. The study was conducted in accordance with the
Declaration of Helsinki (as revised in 2013). This study was approved by the ethics committee of Children's Hospital of Fudan University (No. 2015-170) and informed consent was taken from all the participants.

All neonates, with or without patent ductus arteriosus (PDA) and different types of respiratory support, who were admitted to the Neonatal Department and received the M-mode echocardiography were considered eligible for the study. Exclusion criteria included infants with congenital heart defects (except for small atrial septal defects or a patent foramen ovale), right-to-left shunt PDA, inability to tolerate adhesive skin leads, or the presence of major congenital anomalies. Moreover, infants who were too ill to tolerate additional clinical procedures (including the operation of EC) were excluded.

Several factors influence the potential hemodynamic significance of left-to-right shunting across the ductus arteriosus. Assessment of the hemodynamic significance of PDA (hsPDA) remains difficult. In this study, hsPDA was defined as ductal diameter $\geq 1.4 \mathrm{~mm} / \mathrm{kg}$, or left atrial/ aortic root ratio $(\mathrm{LA} / \mathrm{Ao}) \geq 1.4$ in the group of premature infants (14); for term ones, the ductal diameter was $\geq 2 \mathrm{~mm}$ in infants (6). Furthermore, the respiratory conditions were divided into three groups. These included spontaneous respiration, nasal continuous positive airway pressure (nCPAP), and mechanical ventilation. In addition, the neonates were divided into a low birth weight group (LBW, birth weight $<2,500 \mathrm{~g}$ ) and a non-low birth weight group (non-LBW, birth weight $\geq 2,500 \mathrm{~g}$ ).

\section{Echocardiography}

TTE was performed by employing the Vivid E9 (GE Healthcare, Wauwatosa, WI, USA). All echocardiograms were performed by technicians who had been trained and board-certified in pediatric echocardiography. All measurements were averaged with 3 or more consecutive cardiac cycles. M-mode echocardiography was based on measuring the short axial diameter (D) of the LV. According to record the course of sampling line of M-mode echocardiography through the LV cavity at the level of the chordae tendineae, the short axial D of the $\mathrm{LV}$ at the enddiastole and end-systole were obtained, respectively. This was followed by calculating the LV end-diastolic volume and end-systolic volume, according to the internal algorithm of the echocardiography instrument and using the following equation (15): 


$$
\text { Teichholz equation }=\frac{7 D^{3}}{2.4}+D
$$

$\mathrm{LV}$ cardiac output $(\mathrm{COm})$ was calculated using the formula:

$\mathrm{CO}=$ Stroke Volume * Heart Rate.

The diameter of ductal and shunting direction was measured by the color Doppler and LA/Ao by M-mode echocardiography.

\section{Electrical cardiometry}

An Aesculon ${ }^{\circledR}$ monitor (Osypka Medical, Berlin, Germany) was applied to record EC. Four standard surface electrocardiogram electrodes were placed over the participant's forehead, left side of the neck, left midaxillary line at the level of the xiphoid process, as well as the left thigh. In order to be synchronized with M-mode echocardiography, EC was connected to the participant in advance. During the whole monitoring period, the participant had to be supine and quiet to guarantee a stable signal. The signal was accepted when the signal quality bar was green, and the signal quality was greater than or equal to $80 \%$. The time frequency of each $\mathrm{CO}(\mathrm{COec})$ value generated by EC was 15 seconds. Thus, about $20 \mathrm{COec}$ could be obtained in the whole measurement process. While the technician performed TTE on the participant, another operator completed EC measurements simultaneously. To achieve double blindness, neither the echocardiographic technician nor the EC operator was aware of each other's measurements during the monitoring period. Afterward, another researcher was assigned to collate the average value of COec by using Osypka Medical software, and the value of $\mathrm{COm}$ could be obtained by reviewing the echocardiography records.

\section{Statistical analysis}

The statistical analysis was performed by using SPSS statistical packages, version 22.0 (SPSS Inc., Chicago, IL, USA) and MedCalc.lnk (MedCalc Software, Ostend, Belgium). Continuous variables were presented as median (interquartile range) or mean \pm standard deviation (SD), and categorical variables as percentages. Corresponding values of COec and $\mathrm{COm}$ were tested with a paired $t$-test. The values of COec and COm were converted to $\mathrm{Z}$-scores, according to the following formula:

$$
Z-\text { scores }=\left(C O_{1 \ldots n}-C O_{\text {mean }}\right) / S D_{1 \ldots n}
$$

Pearson's coefficient analyzed the linear correlation of the two methods. Bland-Altman analysis (16) was used to evaluate the consistency between COm Z-scores and COec $\mathrm{Z}$-scores. The results were interpreted by the Bland-Altman index (16), which was defined as the percentage difference between $\mathrm{COm} \mathrm{Z}$-scores and COec Z-scores falling beyond the limit of agreement (LOA). The LOA was defined as bias (the mean difference between COm $Z$-scores and COec Z-scores) $\pm 1.96 \mathrm{SD}$. The Bland-Altman index value did not exceed $5 \%$, indicating a good consistency between the $\mathrm{COm} \mathrm{Z} Z$-scores and $\mathrm{COec} Z$-scores. $\mathrm{CO}$ was presented as $\mathrm{L} / \mathrm{min}$. The $\mathrm{P}$ value $<$ of 0.05 was considered statistically significant.

\section{Results}

\section{Demographic characteristics}

We enrolled 136 participants in this study. A total of 155 pairs of EC and M-mode echocardiography measurements were performed (Figure 1). The clinical characteristics of the participants are shown in Table 1. There were 97 paired CO measurements in the LBW group, 58 paired in the non-LBW group, 46 paired in the hsPDA group, and 109 paired in the non-hsPDA group. There were 96 paired measurements in spontaneous respiration, 25 paired in nCPAP, and 34 paired in mechanical ventilation (11 of which paired in conventional mechanical ventilation, and 23 of which paired in high-frequency oscillatory ventilation).

\section{Correlation between COec Z-scores and COm Z-scores}

The mean values of COec and COm were $(0.47 \pm 0.23) \mathrm{L} / \mathrm{min}$ and $(0.82 \pm 0.33) \mathrm{L} / \mathrm{min}(\mathrm{P}<0.001)$. The correlations between $\mathrm{COec} \mathrm{Z}$-scores and $\mathrm{COm} \mathrm{Z}$-scores were statistically significant in both the overall participant population $(\mathrm{r}=0.601 ; \mathrm{P}<0.001)$ (Figure 2) and other subgroups (Table 2). The correlation coefficients of COec Z-scores and COm $\mathrm{Z}$-scores were similar between hsPDA and non-hsPDA groups $(\mathrm{r}=0.570$ and 0.572 , respectively; both $\mathrm{P}<0.001$ ) (Table 2).

\section{The consistency of COec Z-scores and COm Z-scores}

Figure 3 presents the bias and LOA by Bland-Altman plot. 


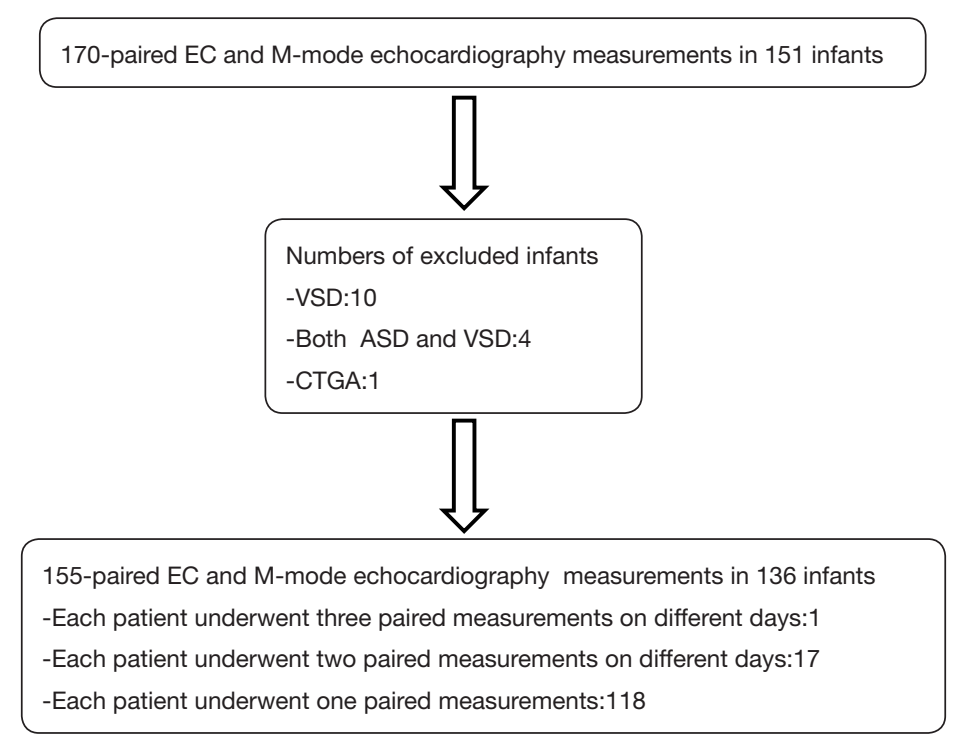

Figure 1 Workflow for patients involved in this trial. ASD, atrial septal defect; CTGA, complete transposition of great arteries; VSD, ventricular septal defect.

Table 1 Clinical characteristics of patients

\begin{tabular}{lc}
\hline Characteristics & Data \\
\hline Gestational age $(\mathrm{w})$, median $[\mathrm{IQR}]$ & $34[29.3-38.8]$ \\
Preterm infants, $\mathrm{n}(\%)$ & $93(68.4)$ \\
Birth weight $(\mathrm{g})$, median $[\mathrm{IQR}]$ & $1873[1,191-2,923]$ \\
Male, $\mathrm{n}(\%)$ & $78(57.4)$ \\
Postnatal day (d), median [IQR] & $6[2-14]$ \\
Body weight (g), median [IQR] & $2065[1,250-2,900]$ \\
Cesarean section, $\mathrm{n}(\%)$ & $74(54.4)$ \\
5 min Apgar score, median $[\mathrm{IQR}]$ & $9[8-10]$ \\
\hline
\end{tabular}

Overall, the Bland-Altman index value was 3.2\% (5/155), indicating a good consistency between COm Z-scores and COec Z-scores. The Bland-Altman index values were $2.1 \%$ (2/97), 3.4\% (2/58), 3.1\% (3/96), 4.0\% (1/25), $2.9 \%(1 / 34)$, $4.3 \%(2 / 46)$, and $3.7 \%(4 / 109)$ in the LBW, non-LBW, spontaneous respiration, nCPAP, mechanical ventilation, hsPDA, and non-hsPDA groups, respectively (Figure 3).

\section{Discussion}

There are a growing number of studies about the comparison of EC and TTE (via VTI) in neonates (6-13). Based on literature reviews, this study analyzed the

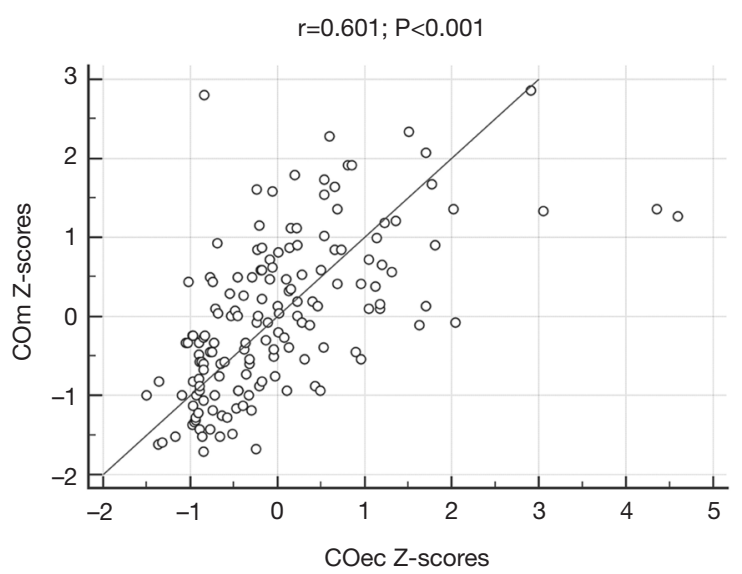

Figure 2 Scatter diagram of COec Z-scores and COm Z-scores.

correlation and consistency of CO values measured with EC and M-mode echocardiography in full-term and preterm neonates.

While multiple techniques exist for ventricular function, each has strengths and weaknesses that should be carefully considered to meet the needs of individual patients. M-mode shortening fraction and ejection fraction are some of the most commonly used methods for LV function (4,5). Estimates of ejection fraction by M-mode, real-time 3-dimensional echocardiography, and Simpson's modified 2-dimensional method were not of significant difference (17). From a practical point of view, M-mode 
Table 2 Correlation between the COec Z-scores and the COm $\mathrm{Z}$-scores in different groups

\begin{tabular}{lcc}
\hline Subgroups & r-value & P value \\
\hline LBW & 0.554 & $<0.001$ \\
non-LBW & 0.377 & 0.004 \\
spontaneous respiration & 0.542 & $<0.001$ \\
nCPAP & 0.431 & 0.031 \\
mechanical ventilation & 0.607 & $<0.001$ \\
hsPDA & 0.570 & $<0.001$ \\
non-hsPDA & 0.572 & $<0.001$ \\
\hline
\end{tabular}

LBW, low birth weight; non-LBW, non-low birth weight; nCPAP, nasal continuous positive airway pressure; hsPDA, hemodynamic significance of the patent ductus arteriosus; non-hsPDA, no hemodynamic significance of the patent ductus arteriosus.

echocardiography has higher temporal resolution and is easy to operate. Therefore, M-mode, rather than color Doppler to measure aortic outflow, measures $\mathrm{CO}$ in our unit. This study found that the mean value of CO measured by M-mode echocardiography $(0.82 \pm 0.33 \mathrm{~L} / \mathrm{min})$ was almost double measured by EC $(0.47 \pm 0.23 \mathrm{~L} / \mathrm{min})$. This result was not surprising, as it was similar to a previous study showing that the Teichholz method overestimated the LV volume by approximately double compared to the real-time 3-dimensional echocardiography and Simpson's modified 2-dimensional method (17). The relative inaccuracy of M-mode echocardiography may be attributed to the geometric modeling of chambers that acquire the single diameter of the $L V$ base, which is incapable of representing the tapered LV morphology (15). This study recognized that the absolute values of $\mathrm{CO}$ measured by $\mathrm{EC}$ and $\mathrm{M}$-mode echocardiography were not interchangeable $(\mathrm{P}<0.001)$.

Furthermore, there was also conflicting evidence on the consistency between EC and TTE (via VTI) (6-13). Since parameters may vary between different measuring methods, the values of $\mathrm{CO}$ were converted to $\mathrm{Z}$-scores, and a good consistency was found between COec Z-scores and $\mathrm{COm} \mathrm{Z}$-scores. This result showed that the distribution of COec was similar to $\mathrm{CO}$ as measured by $\mathrm{M}$-mode echocardiography. Previous studies have shown that hemodynamic parameters by EC are markedly different in neonates of various maturity and provide gestational age and birth weight-based reference for stroke volume and $\mathrm{CO}$ in hemodynamically stable neonates $(9,18)$. Therefore, EC could be used as a supplement for M-mode echocardiography to monitor changes or development trends of $\mathrm{CO}$ in neonates.

The association between EC and TTE (via VTI) has been demonstrated in mixed neonatal populations $(6,8-11)$. This study confirms that EC has a moderate correlation $(\mathrm{r}=0.601 ; \mathrm{P}<0.001)$ with $\mathrm{M}$-mode echocardiography. Boet et al. (9) showed that the correlation of CO measured by EC and TTE (via VTI) remains significant after adjusting for PDA in preterm infants. The study carried out by Song et al. (12) demonstrated that the correlation between EC and TTE (via VTI) is independent of the presence of hsPDA in preterm infants. Noori et al. (6) and Hsu et al. (8) concluded that the consistency between EC and TTE (via VTI) was independent of the confounding effect of hsPDA in neonates, but Torigoe et al. (10) and Blohm et al. (11) presented opposing views. In this study, the correlation coefficients between EC and M-mode echocardiography were similar in hsPDA and non-hsPDA groups. Although the Bland-Altman index values were less than $5 \%$ in hsPDA and non-hsPDA groups, the bias between $\mathrm{COm} \mathrm{Z}$-scores and COec Z-scores was positive in the former group. This result may be related to the different algorithms of $\mathrm{CO}$ between the two methods. As previously mentioned, EC measurements were based on the changes in thoracic impedance, which were mainly affected by the modifications of the volume and erythrocyte orientation in major branches of the aorta. As the main branch of the aorta, the more obvious the left-to-right shunt was, the more significant the hemodynamic significance was, leading to further compensatory increase of the LV output and secondary increase of pulmonary blood flow. Nevertheless, M-mode echocardiography measures the short axial diameter of the LV base before the PDA.

What is more, EC and M-mode echocardiography were compared in participants with diverse respiratory conditions. It was found that $\mathrm{COm} \mathrm{Z} \mathrm{Z}$-scores and $\mathrm{COec}$ $\mathrm{Z}$-scores maintained a good consistency in nCPAP groups. This was in line with Blohm et al.'s (11) findings that nCPAP is not the factor affecting the comparison between EC and M-mode echocardiography in preterm infants. However, the influence of mechanical ventilation on EC and TTE measurements has been controversial in various literature reports. Torigoe et al. (10) and Grollmuss et al. (13) demonstrated that mechanical ventilators did not affect the consistency of the two methods in preterm neonates, but Hsu et al. (8) and Song et al. (12) reached opposite conclusions. In this study, the Bland-Altman index value of the ventilated group was clinically acceptable. 

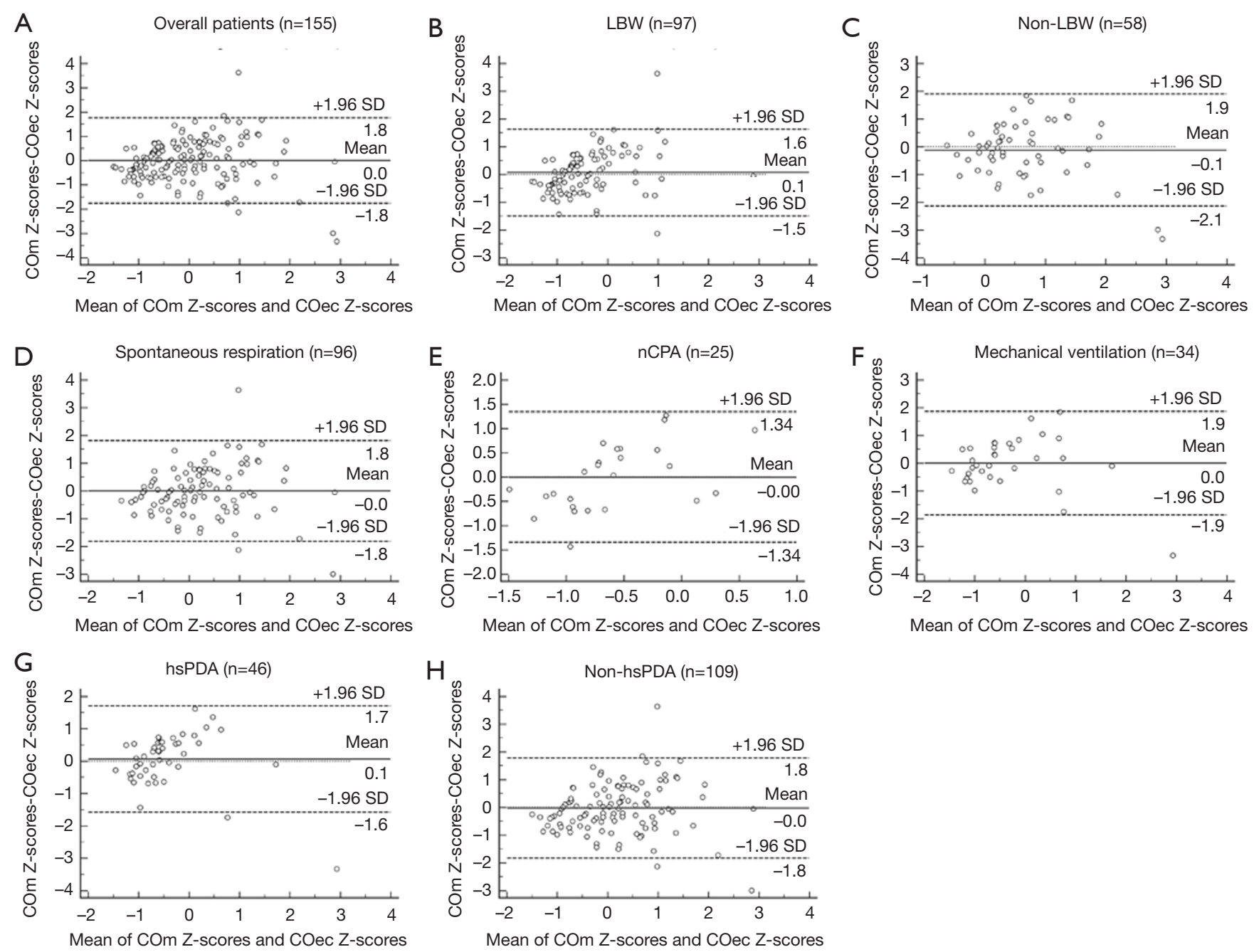

Figure 3 Bland-Altman graphs of COec Z-scores and COm Z-scores. (A) Overall patients; (B) low birth weight (LBW); (C) non-low birth weight (non-LBW); (D) spontaneous respiration; (E) nasal continuous positive airway pressure (nCPAP); (F) mechanical ventilation; (G) hemodynamically significant patent ductus arteriosus (hsPDA); $(\mathrm{H})$ hemodynamically nonsignificant patent ductus arteriosus (non-hsPDA).

Further research is necessary to investigate and analyze the ventilator-associated influence on EC.

\section{Limitations}

There were some limitations in this study. Firstly, as this study's reference method, the M-mode echocardiography is not the gold standard of CO measurement (15). EC also has its limitations in measuring CO. Although this study adjusted the body mass calculation for neonates, the $\mathrm{CO}$ calculations of EC were based on the hemodynamic model in adults. Secondly, the consistency between the two compared methods could be improved by increasing the monitoring interval of measurements (19). Unfortunately, this study only had 1.14 replicates in individual infants. Thirdly, EC is easily applied, but studies of its value in clinical work are needed. Hemodynamic parameters, such as $\mathrm{CO}$, total thoracic fluid (TFC), and stroke volume variation (SVV) provided by EC could be combined with other clinical data to detect hemodynamic abnormalities as well as assess the therapeutic effect and prognosis $(20,21)$.

\section{Conclusions}

There was a significant correlation between EC and M-mode echocardiography, which could not be altered by 
different ventilation supports or hsPDA in neonates. As a continuous, non-invasive monitoring method, EC can reflect the distribution of $\mathrm{CO}$ in $\mathrm{M}$-mode echocardiography for neonates, although it is not comparable with M-mode echocardiography.

\section{Acknowledgments}

We would like to thank the patients and their parents for participating in this study, and the neonatal staff at Children's Hospital of Fudan University for their assistance. Funding: This study was supported by Big Data and Artificial intelligence Research Funding project of clinical Research Center (No. 2020DSJ13).

\section{Footnote}

Reporting Checklist: The authors have completed the STROBE reporting checklist. Available at https://dx.doi. org/10.21037/tp-21-20

Data Sharing Statement: Available at https://dx.doi. org/10.21037/tp-21-20

Conflicts of Interest: All authors have completed the ICMJE uniform disclosure form (available at https://dx.doi. org/10.21037/tp-21-20). The authors have no conflicts of interest to declare.

Ethical Statement: The authors are accountable for all aspects of the work in ensuring that questions related to the accuracy or integrity of any part of the work are appropriately investigated and resolved. The study was conducted in accordance with the Declaration of Helsinki (as revised in 2013). This study was approved by the ethics committee of Children's Hospital of Fudan University (No. 2015-170) and informed consent was taken from all the participants.

Open Access Statement: This is an Open Access article distributed in accordance with the Creative Commons Attribution-NonCommercial-NoDerivs 4.0 International License (CC BY-NC-ND 4.0), which permits the noncommercial replication and distribution of the article with the strict proviso that no changes or edits are made and the original work is properly cited (including links to both the formal publication through the relevant DOI and the license).
See: https://creativecommons.org/licenses/by-nc-nd/4.0/.

\section{References}

1. Soleymani S, Borzage M, Noori S, et al. Neonatal hemodynamics: monitoring, data acquisition and analysis. Expert Rev Med Devices 2012;9:501-11.

2. Summers RL, Shoemaker WC, Peacock WF, et al. Bench to bedside: electrophysiologic and clinical principles of noninvasive hemodynamic monitoring using impedance cardiography. Acad Emerg Med 2003;10:669-80.

3. Kluckow M, Seri I, Evans N. Functional echocardiography: an emerging clinical tool for the neonatologist. J Pediatr 2007;150:125-30.

4. Lai WW, Geva T, Shirali GS, et al. Guidelines and standards for performance of a pediatric echocardiogram: a report from the Task Force of the Pediatric Council of the American Society of Echocardiography. J Am Soc Echocardiogr 2006;19:1413-30.

5. Mertens L, Seri I, Marek J, et al. Targeted neonatal echocardiography in the neonatal intensive care unit: practice guidelines and recommendations for training:. Eur J Echocardiogr 2011;12:715-36.

6. Noori S, Drabu B, Soleymani S, et al. Continuous non-invasive cardiac output measurements in the neonate by electrical velocimetry: a comparison with echocardiography. Arch Dis Child Fetal Neonatal Ed 2012;97:F340-3.

7. Grollmuss O, Demontoux S, Capderou A, et al. Electrical velocimetry as a tool for measuring cardiac output in small infants after heart surgery. Intensive Care Med 2012;38:1032-9.

8. Hsu KH, Wu TW, Wu IH, et al. Electrical Cardiometry to Monitor Cardiac Output in Preterm Infants with Patent Ductus Arteriosus: A Comparison with Echocardiography. Neonatology 2017;112:231-7.

9. Boet A, Jourdain G, Demontoux S, et al. Stroke volume and cardiac output evaluation by electrical cardiometry: accuracy and reference nomograms in hemodynamically stable preterm neonates. J Perinatol 2016;36:748-52.

10. Torigoe T, Sato S, Nagayama Y, et al. Influence of patent ductus arteriosus and ventilators on electrical velocimetry for measuring cardiac output in very-low/low birth weight infants. J Perinatol 2015;35:485-9.

11. Blohm ME, Obrecht D, Hartwich J, et al. Impedance cardiography (electrical velocimetry) and transthoracic echocardiography for non-invasive cardiac output 
monitoring in pediatric intensive care patients: a prospective single-center observational study. Crit Care 2014;18:603.

12. Song R, Rich W, Kim JH, et al. The use of electrical cardiometry for continuous cardiac output monitoring in preterm neonates: a validation study. Am J Perinatol 2014;31:1105-10.

13. Grollmuss O, Gonzalez P. Non-invasive cardiac output measurement in low and very low birth weight infants: a method comparison. Front Pediatr 2014;2:16.

14. El Hajjar M, Vaksmann G, Rakza T, et al. Severity of the ductal shunt: a comparison of different markers. Arch Dis Child Fetal Neonatal Ed 2005;90:F419-22.

15. Teichholz LE, Kreulen T, Herman MV, et al. Problems in echocardiographic volume determinations: echocardiographic-angiographic correlations in the presence of absence of asynergy. Am J Cardiol 1976;37:7-11.

16. Bland JM, Altman DG. Statistical methods for assessing agreement between two methods of clinical measurement.
Lancet 1986;1:307-10.

17. Tidholm A, Westling AB, Höglund K, et al. Comparisons of 3-, 2-dimensional, and M-mode echocardiographical methods for estimation of left chamber volumes in dogs with and without acquired heart disease. J Vet Intern Med 2010;24:1414-20.

18. Hsu KH, Wu TW, Wang YC, et al. Hemodynamic reference for neonates of different age and weight: a pilot study with electrical cardiometry. J Perinatol 2016;36:481-5.

19. Whitley E, Ball J. Statistics review 4: sample size calculations. Crit Care 2002;6:335-41.

20. Hsu KH, Wu TW, Wu IH, et al. Baseline cardiac output and its alterations during ibuprofen treatment for patent ductus arteriosus in preterm infants. BMC Pediatr 2019;19:179.

21. Butz J, Shan Y, Samayoa A, et al. The utility of impedance cardiography in hemodynamic monitoring of patients with sepsis. Trauma Surg Acute Care Open 2019;4:e000349.
Cite this article as: $\mathrm{Xu} \mathrm{SH,} \mathrm{Zhang} \mathrm{J,} \mathrm{Zhang} \mathrm{Y,} \mathrm{Zhang} \mathrm{P,} \mathrm{Cheng}$ GQ. Non-invasive cardiac output measurement by electrical cardiometry and M-mode echocardiography in the neonate: a prospective observational study of 136 neonatal infants. Transl Pediatr 2021;10(7):1757-1764. doi: 10.21037/tp-21-20 